\title{
回转筒颗粒表面流的分子动力学模拟和连续理论
}

\author{
李水清 ${ }^{(}$姚 强 ${ }^{(}$陈 冰 ${ }^{(1}$ 张 宣 $^{(1)}$ Ding Y. D. ${ }^{(2)}$
}

(1) 清华大学热能工程系, 北京 100084; (2) 利兹大学化学工程系, 利兹 LS2 9JT, 英国. E-mail: lishuiqing@mail.tsinghua.edu.cn)

\begin{abstract}
摘要 在考虑颗粒非弹性接触、滑动摩擦和滚动摩擦基础上发展了分子动力学模拟的算法, 实现了中高 速 $(F r=0.1 \sim 0.2)$ 回转筒内颗粒流的离散模拟. 回转筒内颗粒流由表面活性层和下部柱塞流区组成, 颗粒 在活性层的停留时间约为柱塞流区的 $1 / 3 \sim 1 / 2$, 对称线上活性层和柱塞流区的厚度比为 $0.57 \sim 0.61$, 因而 推断颗粒流动处于 Rolling-Cascading 过渡模式. 对称线上 MD 模拟的速度分布与正电子放射性测量实 验结果十分吻合. 在模拟和实验结果基础上发展了连续理论: 柱塞流区内颗粒运动并非完全随着筒壁 刚体转动, 而是存在着塑性蠕变, 这种速度变化过程符合指数函数规律; 而活性层内颗粒流动则符合简 单的 Couette 切变流动分布. 最后探讨了颗粒温度和颗粒相对浓度分布的内在机理.
\end{abstract}

关键词 颗粒流 回转筒 分子动力学模拟 连续理论

颗粒体(granular material)是一些离散的固相颗粒 形成的聚集体, 在没有外界扰动下是静止的, 当受到 外界剪切、振动、转动或流体扰动的作用下, 颗粒体 逐渐呈现出类似液体行为, 甚至在料层表面形成连 续的颗粒流动 ${ }^{[1,2]}$. 这种颗粒流与传统气体或液体为 代表的分子流最大区别在于: (i ) 分子运动的状态 取决于热力学温度, 而宏观的颗粒在重力作用下几 乎没有热能效应, 其状态决定于外加力的作用或能 量, 为统一表示不同形式力的作用, 可引入颗粒温度 (即颗粒脉动速度均方差)的概念来表征颗粒的状态; (ii) 与分子之间的弹性碰撞不同, 颗粒之间非弹性 接触使得颗粒体是一个强耗散性系统, 为维持颗粒 温度不变, 必须有外加能量不断地输入到系统, 回转 筒和振动床就是两种最具代表性的实例; (iii) 对于 分子流, 任何一个微元控制容积体内都有足够数量 的分子来满足连续介质假设, 而且其压力和温度参 数的平均值足够精确, 然而对于颗粒流, 其微元控制 容积体的尺度可能和颗粒尺度在一个数量级上, 因 而直接采用连续介质理论开展研究的难度较大 ${ }^{[3]}$.

20 世纪 90 年代以来颗粒流研究已逐渐成为国际 上工程和物理科学研究的热点之一, 相关研究主要 集中在 3 个方面: (i ) 没有外力、只有重力作用的体 系, 如倾斜平面、坚直槽道和自然堆积斜面上的颗粒 流动, 相应的连续性动力学理论(kinetic theory)和离 散分子动力学模拟(molecular dynamics, MD)的研究 都得以迅速发展 ${ }^{[4,5]}$; (ii ) 边界周期性运动和重力共 同作用的振动料床, 过去 10 年加州理工学院Hunt研 究小组 ${ }^{[6]}$ 也使得其理论体系相对成熟; (iii) 壁面转动
和重力共同作用的回转料床, 如工程中普遍存在的 混合器、造粒机和回转窑等 ${ }^{[7]}$, 尽管其应用背景尤为 重要而日益受到重视, 但其相应基础研究仍在发展 和完善之中 ${ }^{[8,9]}$.

分子动力学模拟(MD) 是非连续体所适用的数值 方法, MD以颗粒体的接触力学理论为基础, 结合牛 顿第二定律及显性时间积分法来描述每一个颗粒在 单个时间步长的运动行为 $[10,11]$. 本文从分子动力学 模拟思路出发, 在软球模型基础上首次补充了转动 惯量方程的滚动摩擦一阶修正项, 从而实现回转筒 内颗粒表面流的模拟, 模拟的速度场与文献中实验 结果相吻合, 最后发展了描述颗粒速度分布的连续 理论并和MD结果进行了比较.

\section{1 分子动力学模拟方法}

\section{1 牛顿定律方程式}

回转筒内任一颗粒的运动分为平动和转动, 采 用牛顿第二定律分别表示为

$$
\begin{gathered}
m_{i} \frac{\mathrm{d}^{2} x_{i}}{\mathrm{~d} t^{2}}=m_{i} \frac{\mathrm{d} v_{i}}{\mathrm{~d} t}=\sum_{j}\left(F_{i j}^{n}+F_{i j}^{s}+m_{i} g\right), \\
I_{i} \frac{\mathrm{d} \bar{\omega}_{i}}{\mathrm{~d} t}=\sum_{j}\left(r_{i} \times F_{i j}^{s}-M_{i j}\right),
\end{gathered}
$$

式中 $x_{i}, v_{i}, \bar{\omega}_{i}$ 和 $I_{i}$ 分别是颗粒的位移、平动速度、 转动角速度和转动惯量. $F_{i j}^{n}$ 和 $F_{i j}^{s}$ 分别是颗粒 $i$ 和 $j$ 之间的法向和切向作用力(如图 1).

\section{2 颗粒间接触力模型}

如图 1 , 7 颗粒间法向接触力 $\left(F_{i j}^{n}\right)$ 包含弹性力及耗 


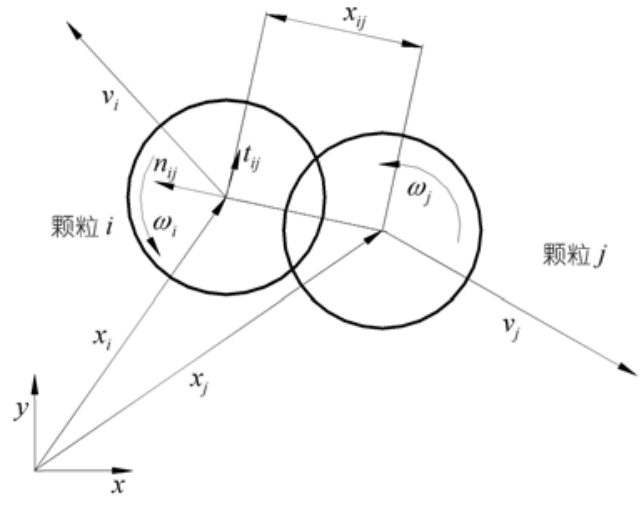

(a)
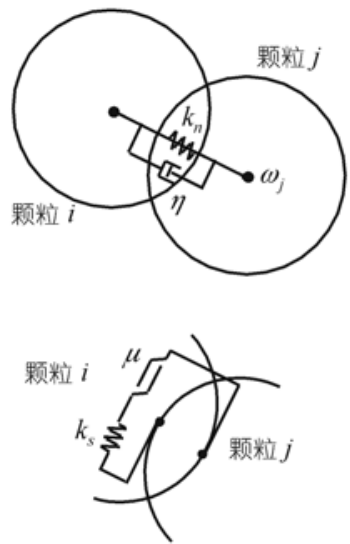

(b)

图 1 两个颗粒接触的示意图

(a) 参数变量; (b) 法向和切向接触力

散力两项. 与传统MD方法将分子视为弹性碰撞不同, 本文将颗粒间碰撞视为存在摩擦力的非弹性碰撞, 即软球模型. 根据Hook定律, $F_{i j}^{n}$ 中弹性力与两个颗 粒 $i$ 和 $j$ 之间重叠量(overlap)成线性关系 [12], 而耗散力 可假定与颗粒相对速度成线性关系, 则 $F_{i j}^{n}$ 可表示为

$$
F_{i j}^{n}=\tilde{Y} r_{\mathrm{eff}}\left(r_{i}+r_{j}-\left|x_{i j}\right|\right) n_{i j}-\gamma_{n} m_{\mathrm{eff}}\left(v_{i j} \cdot n_{i j}\right) n_{i j},
$$

其中 $\tilde{Y}$ 是颗粒杨氏弹性模量, $\gamma_{n}$ 是法向动耗散系数; $r_{\mathrm{eff}}$ 是颗粒有效半径, 且满足 $r_{\mathrm{eff}}=r_{i} r_{j} /\left(r_{i}+r_{j}\right), m_{\mathrm{eff}}$ 是有效质量且满足 $m_{\mathrm{eff}}=m_{i} m_{j} /\left(m_{i}+m_{j}\right) ; x_{i j}=x_{i}-$ $x_{j}$ 是颗粒之间距离; $n_{i j}$ 是由颗粒 $j$ 指向颗粒 $i$ 的单位 矢量, 即 $n_{i j}=x_{i j} /\left|x_{i j}\right|$. 颗粒相对速度 $v_{i j}$ 可表示为

$$
v_{i j}=v_{i}-v_{j}-\left(r_{i} w_{i}+r_{j} w_{j}\right) \times n_{i j} .
$$

颗粒切向接触力可假设只包含弹性力一项 ${ }^{[2,13,14]}$, 但弹性力大小不会超过最大静摩擦力, 如果弹性力 超过最大静摩擦力, 则发生滑动摩擦, 根据理解库仑 屈服定律, 以摩擦力 $\mu_{s}\left|F_{i j}^{n}\right|$ 代替弹性力, 但方向仍维 持原弹性力的方向, 如下式所示:

$$
F_{i j}^{s}=-\operatorname{sgn}\left(\delta_{s}\right) \min \left(k_{s}\left|\delta_{s}\right|, \mu_{s}\left|F_{i j}^{n}\right|\right),
$$

其中 $k_{s}$ 为切向力弹性常数, 和法向力弹性常数满足 关系 $k_{s}=(0.67-1.0) k_{n}=(0.67-1.0) \tilde{Y}_{\mathrm{eff}}{ }^{[14]} ; \mu_{s}$ 为滑动 摩擦系数; $\delta_{s}$ 为颗粒 $i$ 和 $j$ 从初始接触时刻开始的切向 位移, 表示为

$$
\delta_{s}=-\int v_{i j}^{s} \mathrm{~d} t=-\int v_{i j} \cdot s_{i j} \mathrm{~d} t,
$$

式中 $s_{i j}$ 是颗粒 $i$ 和 $j$ 接触时的单位切向矢量.

\section{3 滚动摩擦阻力矩的修正项}

颗粒之间滚压接触过程中还会产生不同形式的 能量消耗, 称之为滚动摩擦, 黏弹性颗粒的滚动摩擦 主要取决于颗粒堆积的变形损失, 而非颗粒表面效 应 ${ }^{[15]}$. 因此, 我们假设滚动摩擦大小仅与颗粒堆积 所呈现的黏性耗散有关(图 2(b)), 即颗粒表面效应导 致的损失忽略不计. 根据图 2(a)所示, 滚动摩擦阻力 矩可以表示为

$$
M_{i j}=-\int_{s_{a}}\left(r-r_{O^{\prime}}\right) \times F_{n} \mathrm{~d} s,
$$

式中 $S_{a}$ 为接触平面上圆形轮廓线, 且 $r_{O^{\prime}}=\left(0, y_{0}, 0\right)$. Brilliantov和Pöschel积分上述方程有 [14]

$$
M_{i j}=-\mu_{r}\left(r_{i} \bar{\omega}_{i}\right)\left|F_{i j}^{n}\right| .
$$

为正确求解方程 (2), 假设滚动摩擦只从数值上减少 转动力矩, 而不改变方向, 则其限制为

$$
\left|M_{i}\right|=\left|\sum_{j} M_{i j}\right| \leqslant I_{i} \bar{\omega}_{i} / \Delta t .
$$

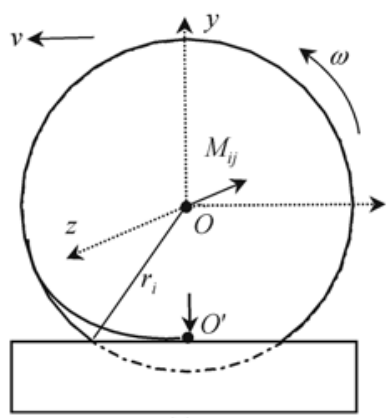

(a)

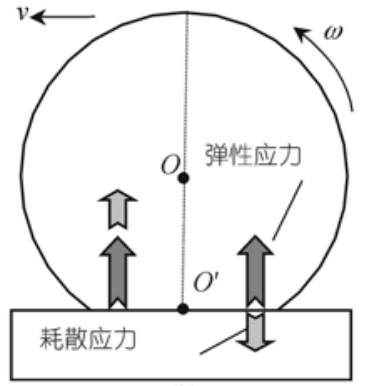

(b)
图 2 黏弹性球状颗粒在硬面上的滚压摩擦示意图 (a) 滚动阻力矩; (b) 能量耗散

$1.4^{10}$ 具体计算算法 
颗粒间作用力计算的实现 联立方程(1)，(2)与 (3), (5), (9)可以求得任意时刻颗粒的位置乃至速度场. 如果所有颗粒对之间作用力都统计, 即采用全颗粒 对计录法(all-pairs method)时, 颗粒数 $N_{m}$ 的计算总 步数是 $O\left(N_{m}^{2}\right)$. 为降到 $O\left(N_{m}\right)$ 量级, 本文采用邻居 列表法(neighbor-list method). 该方法思想为: 任意时 刻某一颗粒速度是有限的, 所以 $\Delta t$ 内颗粒运动轨迹 所覆盖范围是一个以其为球心的球体(假设颗粒速度 方向任意). 换言之, 在下一个 $\Delta t$ 时刻, 该颗粒只能与 在这个球体范围内的颗粒发生碰撞. 首先将整个计 算空间划分为二维空间许多相邻的小室，每个颗粒 散布在各个小室内. 这样计算颗粒间相互作用力时 仅考虑该颗粒所处小室内其他颗粒以及相邻小室内 的颗粒与该颗粒的作用力, 大大缩减计算量. 当颗粒 跨越小室边界时, 则要更新该邻居列表 ${ }^{[10]}$.

积分过程计算的实现 微分方程(1)和(2)的积分 可通过预估-校正法(predictor-corrector method)实现. 对于预估阶段满足

$$
\begin{aligned}
& v_{i}(t+\Delta t / 2)=v_{i}(t)+(\Delta t / 2) \cdot a_{i}(t), \\
& x_{i}(t+\Delta t)=x_{i}(t)+\Delta t \cdot v_{i}(t+\Delta t / 2),
\end{aligned}
$$

同时校正阶段则有

$$
v_{i}(t+\Delta t)=v_{i}(t+\Delta t / 2)+(\Delta t / 2) \cdot a_{i}(t+\Delta t) .
$$

\section{5 模型参数的选择}

假设两个颗粒 $i$ 和 $j$ 发生碰撞简单系统，则根据 (1)式, 两个颗粒之间位移差表示为

$$
\mathrm{d}^{2}\left(x_{i}-x_{j}\right) / \mathrm{d} t^{2}=F_{i j}^{n} / m_{\text {eff }} .
$$

$\delta=\left(r_{i}+r_{j}-\left(x_{i}-x_{j}\right)\right)$ 是两个颗粒之间重叠量, 代入 Hook 定律则颗粒碰撞非线性方程为

$$
\frac{\mathrm{d}^{2} \delta}{\mathrm{d} t^{2}}+\gamma_{n} \frac{\mathrm{d} \delta}{\mathrm{d} t}+\frac{\tilde{Y} r_{\text {eff }}}{m_{\text {eff }}} \delta=0(\delta>0) .
$$

方程(13)和(14)为标准线性阻尼作用的一维简谐振动 方程. 边界条件为 $t=0, \delta=0, \mathrm{~d} \delta / \mathrm{d} t=v_{0}$. 假设即 弱阻尼情况下, 设定 $\beta=\gamma_{n} / 2, \bar{\omega}_{0}^{2}=\tilde{Y} r_{\text {eff }} / m_{\text {eff }}$ 和 $\bar{\omega}_{\eta}^{2}=\bar{\omega}_{0}^{2}-\left(\gamma_{n} / 2\right)^{2}$, 方程分析解为

$$
\begin{aligned}
& \delta(t)=\frac{v_{0}}{w_{\eta}} \mathrm{e}^{-\beta t} \sin \left(w_{\eta} t\right)=\frac{v_{0}}{w_{\eta}} \mathrm{e}^{-\gamma_{n} t / 2} \sin \left(w_{\eta} t\right), \\
& \mathrm{a}(t)=\frac{\mathrm{d}^{2} \delta(t)}{\mathrm{d} t^{2}}=\frac{v_{0}}{w_{\eta}} \mathrm{e}^{-\gamma_{n} t / 2}\left[-\gamma_{n} / 2 \sin \left(w_{\eta} t\right)+w_{\eta} \cos \left(w_{\eta} t\right)\right] .
\end{aligned}
$$

此外, 表征非弹性碰撞程度的恢复系数定义为 $e=\exp \left(-\gamma_{n} \pi / \bar{\omega}_{\eta}\right)$.

图 3 和 4 是两个球发生水平对心碰撞时两颗粒之 间重叠量和加速度随碰撞时间的变化 $\left(d_{\mathrm{p}}=3.0 \mathrm{~mm}\right.$, $v_{0}=0.2 \mathrm{~m} / \mathrm{s}$ ). 首先对于弹性系数 $k_{n}=\tilde{Y}_{\text {eff }}$ 的选取, Weber ${ }^{[16]}$ 对其做了简要的综述, 近 10 位学者选用 800 $\mathrm{kg} / \mathrm{s}^{2}$ 这个值附近, 且认为该量纲内 $k_{n}$ 值对计算结果 影响不大, 因此本文选取杨氏弹性模量 $\tilde{Y}=5 \times 10^{5}$ $\mathrm{kg} /\left(\mathrm{m} \cdot \mathrm{s}^{2}\right)$, 相应弹性系数为 $750 \mathrm{~kg} / \mathrm{s}^{2}$. 这不仅与 Weber推荐的范围吻合, 也与Peters在其综述中推荐 $\tilde{Y}=5 \times 10^{5} \mathrm{~kg} /\left(\mathrm{m} \cdot \mathrm{s}^{2}\right)$ 相符合 $\stackrel{[13]}{\text {. }}$.

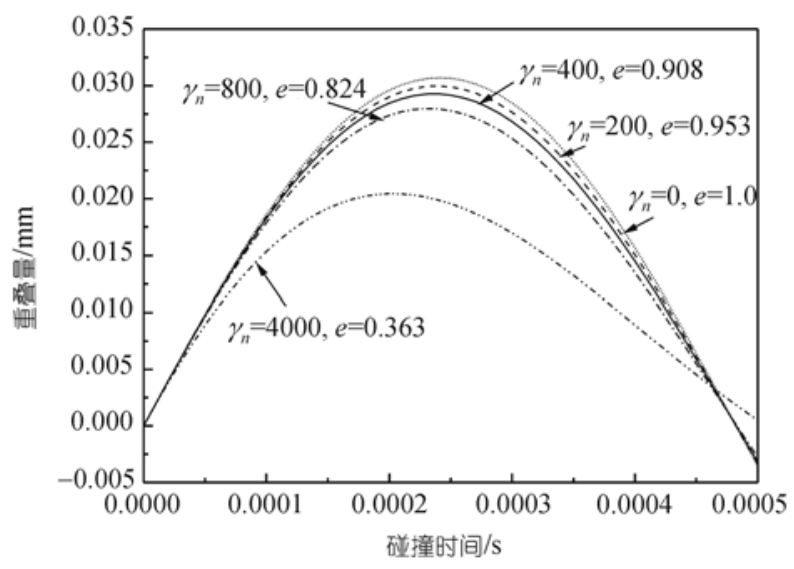

图 3 两球对心碰撞重叠量随碰撞时间的变化

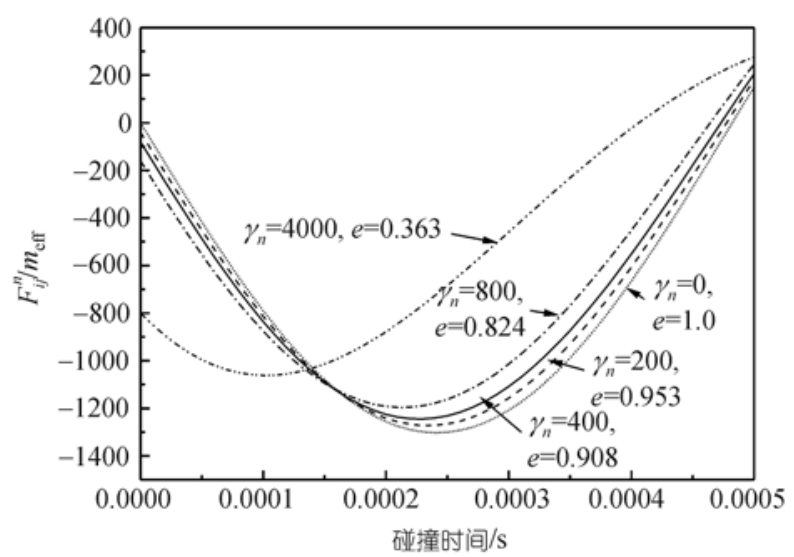

图 4 两球对心碰撞法向加速度随碰撞时间的变化

相对于弹性系数 $k_{n}$, 对耗散系数的选取已有研 究尚未形成共识. 本文基于 $m_{\mathrm{eff}}$ 导出了无量纲耗散 系数 $\gamma_{n}$ (如图 3), 发现当 $\gamma_{n}$ 从 0 增加到 $800 \mathrm{~s}^{-1}$ 时, 碰 撞恢复系数从 1 减小到 0.824 , 最大重叠量 $\delta_{\text {max }}$ 差别也 仅有 $10 \%$. 但是当 $\gamma_{n}$ 增加到 $4000 \mathrm{~s}^{-1}$ 时, 恢复系数骤 减到 0.363 , 此时 $\delta$ 随时间变化曲线的形状和大小都与 e $>0.824$ 的 4 个正况有根本不同. 同样地由图 $4, \gamma_{n}$ 
在 $0 \sim 800 \mathrm{~s}^{-1}(e>0.824)$ 的 4 个工况下颗粒法向加速度 随时间变化曲线的形状和大小相当接近, 各工况差 别小于 $15 \%$, 而 $\gamma_{n}=4000 \mathrm{~s}^{-1}$ 工况则差别十分大. 特 别注意当 $\gamma_{n}$ 较大 ( $e$ 较小)时, 初始接触时作用力偏离 零点与物理实际不符, 说明本文线性碰撞仅适用于 恢复系数较大的物料. 而本文采用玻璃球碰撞后恢 复特性较好, 因此选取 $\gamma_{n}=400 \mathrm{~s}^{-1}, e=0.908$ 是合理的 [17].

\section{2 结果与讨论}

正电子放射性测量技术(PEPT) 是Birmingham大 学开发的颗粒流场无扰测量手段, Parker 等人 [18]和 Ding等人 ${ }^{[19]}$ 先后采用该技术首次实现了回转筒内颗 粒流场的测量. 本文研究的模拟工况取自 Parker等人 的实验工况, 已有结论认为回转筒内颗粒轴向混合 远小于径向混合，为节省时间本文采用基于拟三维 床思想的二维模拟 ${ }^{[20]}$ ，即筒体长度等于一个颗粒直 径长, 颗粒转动惯量计算采用圆球而非圆盘的公式, 即 $2 / 5 m r_{\mathrm{p}}^{2}$, 详细参数列于表 1 .
图 5 所示是 $42 \mathrm{r} / \mathrm{min}$ 转速时不同时间下MD模拟 的颗粒流动情况. 根据单颗粒示踪可把颗粒流动模 式分为两个区域: 上部表面区内，颗粒沿倾斜表面做 滚落运动, 速度略快, 停留时间约 $0.3 \sim 0.4 \mathrm{~s}$, 因而称 之为活性层(active layer); 下部区域中, 颗粒随着筒 壁转动做回转运动, 停留时间大约 $0.8 \mathrm{~s}$, 该区被其他 学者称之为柱塞流区(plug-flow region) ${ }^{[7,19,21,22]}$. 般地, 随着Froude数 $\left(F r=\omega^{2} R / g\right)$ 提高, 回转筒内颗 粒流将经历滑移(slipping), 塌落(slumping), 滚动 (rolling), 泻落 (cascading), 抛落(cataracting)和离心运 动等模式，其中Rolling和Cascading模式的特征都是 由活性层和柱塞流区组成, 细微不同在于Cascading 模式下料床表面的线性度没有Rolling模式好 ${ }^{[23]}$. 图 5 工况中 Fr数是 0.098 , 据文献 [22,23]中 Fr准则判据, 可视为Rolling-Cascading过渡区，MD模拟结果表明 在部分时刻料床从线形变为明显的拱形，符合过渡 模式的特征, 也与Orpe和Kharkar ${ }^{[24]}$ 的实验结果相符

\subsection{MD 模拟结果分析}

表 1 模拟参数工况汇总表

\begin{tabular}{cccccc}
\hline 筒体半径 $R$ & 筒体长度 $L$ & 颗粒填充率 $\Gamma$ & 颗粒表面到圆心距离 $h_{0}$ & 颗粒直径 $d_{\mathrm{p}}$ & 颗粒密度 $\rho_{\mathrm{p}}$ \\
\hline $50 \mathrm{~mm}$ & $3 \mathrm{~mm}$ & $36 \%$ & $11 \mathrm{~mm}$ & $3 \mathrm{~mm}$ & $2600 \mathrm{~kg} / \mathrm{m}^{3}$ \\
\hline \hline 杨氏弹性模量 $\tilde{Y}$ & 法向弹性系数 $k_{n}$ & 切向弹性系数 $k_{s}$ & 颗粒间滑动摩擦系数 $\mu_{\mathrm{p}}$ & 壁面滑动摩擦系数 $\mu_{\mathrm{w}}$ & 壁面滚动摩擦系数 $\mu_{\mathrm{rp}}$ \\
\hline $5 \times 10^{5} \mathrm{~kg} /\left(\mathrm{m} \cdot \mathrm{s}^{2}\right)$ & $750 \mathrm{~kg} / \mathrm{s}^{2}$ & $1.0 k_{n}$ & 0.4 & 0.4 & 0.4 \\
\hline
\end{tabular}
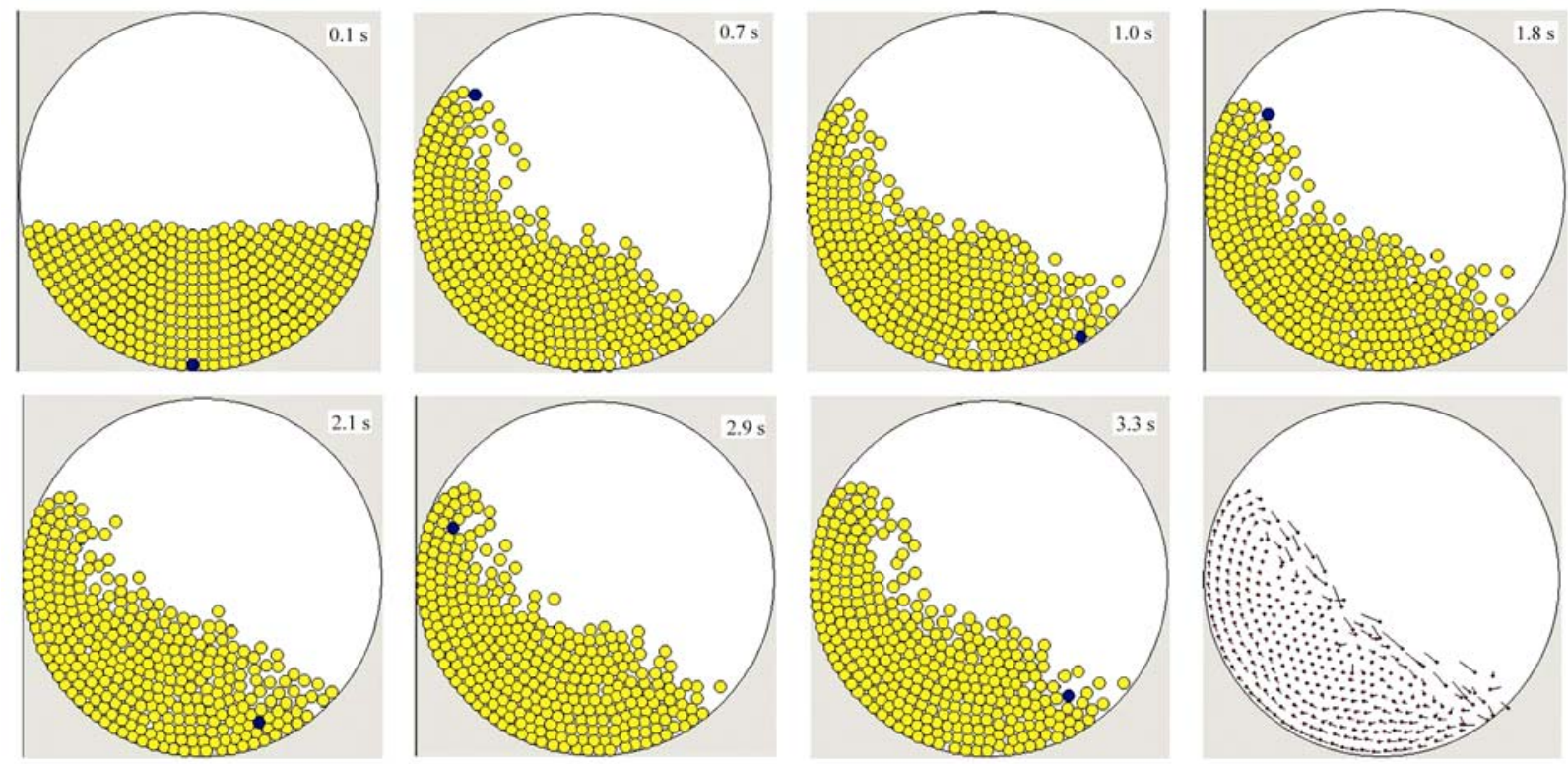

图 5 回转筒内颗粒流场的 $\mathrm{MD}$ 模拟结果 $\left(n=42 \mathrm{r} / \mathrm{min}, d_{\mathrm{p}}=3 \mathrm{~mm}, D=100 \mathrm{~mm}\right)$ 
合. Yang等人 ${ }^{[25]}$ 针对该工况模拟研究却得出该区仍 处于纯Rolling模式的结论，但纯Rolling模式 Fr 数上 限是 0.02 , 远小于 0.098 . 而且, Boateng [22]得出 $5 \times 10^{-4}<\mathrm{Fr}<1.2 \times 10^{-3}$ 的Rolling模式下，活性层厚度 仅是柱塞流区 $20 \%$ ，因而活性层停留时间相对于柱 塞流区停留时间可以忽略, $\mathrm{Li}$ 等人 ${ }^{[7]}$ 在这个Fr范围内 也证实了该结论. 这与本文研究所得的两个区停留 时间仅相差 2 3 倍不同, 因此可间接地得出图 5 中颗 粒流模式应是Rolling-Cascading过渡区.

\section{$2.2 \mathrm{MD}$ 模拟值和实验值的比较}

MD 微观模型适用性的验证依赖于从模拟结 果中抽取出具有代表性的宏观特征. Ding等人 [19]、 Kharkar等人 [21]和Boateng ${ }^{[22]}$ 发展了回转筒内颗粒流 的连续理论(continuum model), 指出中线速度分布假 设是质量和动量守恒方程求解的前提. 图 6 所示是筒 内颗粒流区划分和对称线速度分布, 而对称线速度 分布即可视为验证各理论的宏观特征.

图 7 和 8 示出两种转速下对称线上颗粒周向速度 的PEPT实验值和MD模拟均值的比较. 柱塞流区二 者吻合较好, 在上部活性层二者也基本吻合, 但在接 近料床表面处二者存在一定差别. $42 \mathrm{r} / \mathrm{min} 工$ 况表面 处MD模拟值比实验值大 $27.4 \%, 65 \mathrm{r} / \mathrm{min}$ 时则大 $27.5 \%$. 究其原因在于实验结果来自Parker等人 ${ }^{[18]}$ 的

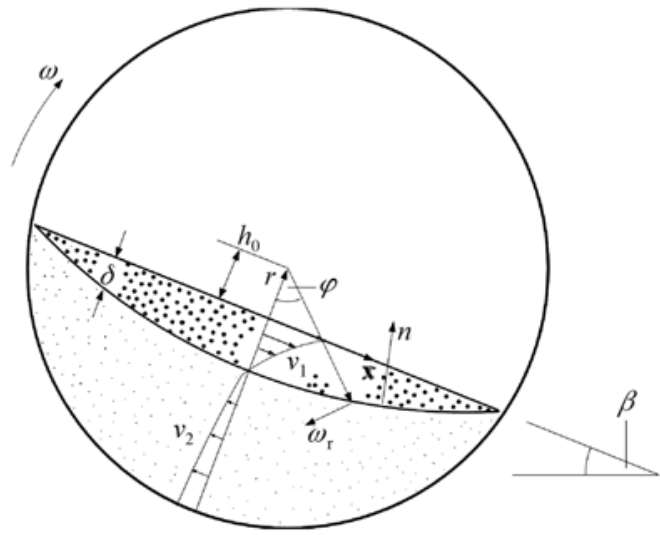

图 6 回转筒流区划分和速度分布

三维测量, 而模拟结果是单层颗粒的拟三维模拟, 下 部区域由于颗粒主要发生的是随着筒体的转动, 颗 粒间紧密接触使得轴向扩散几乎不会发生, 因此该 区吻合较好; 但在表面颗粒之间接触较少, 会发生自 由滚落运动, 多层三维床颗粒碰到其他粒子的概率 高于单层二维床, 从而会降低滚落速度, 因此MD模 拟值要略大于实验值. Maneval等人 ${ }^{[26]}$ 采用磁共振成 像技术测量发现二维床和三维床对称线上速度分布 几乎一致, 仅在料床表面存在着 $30 \%$ 40\%的差别, 这也部分说明了图 7 和 8 中MD模拟的合理性.

图 7 和 8 中 MD 的成功模拟与滚动摩擦阻力矩修

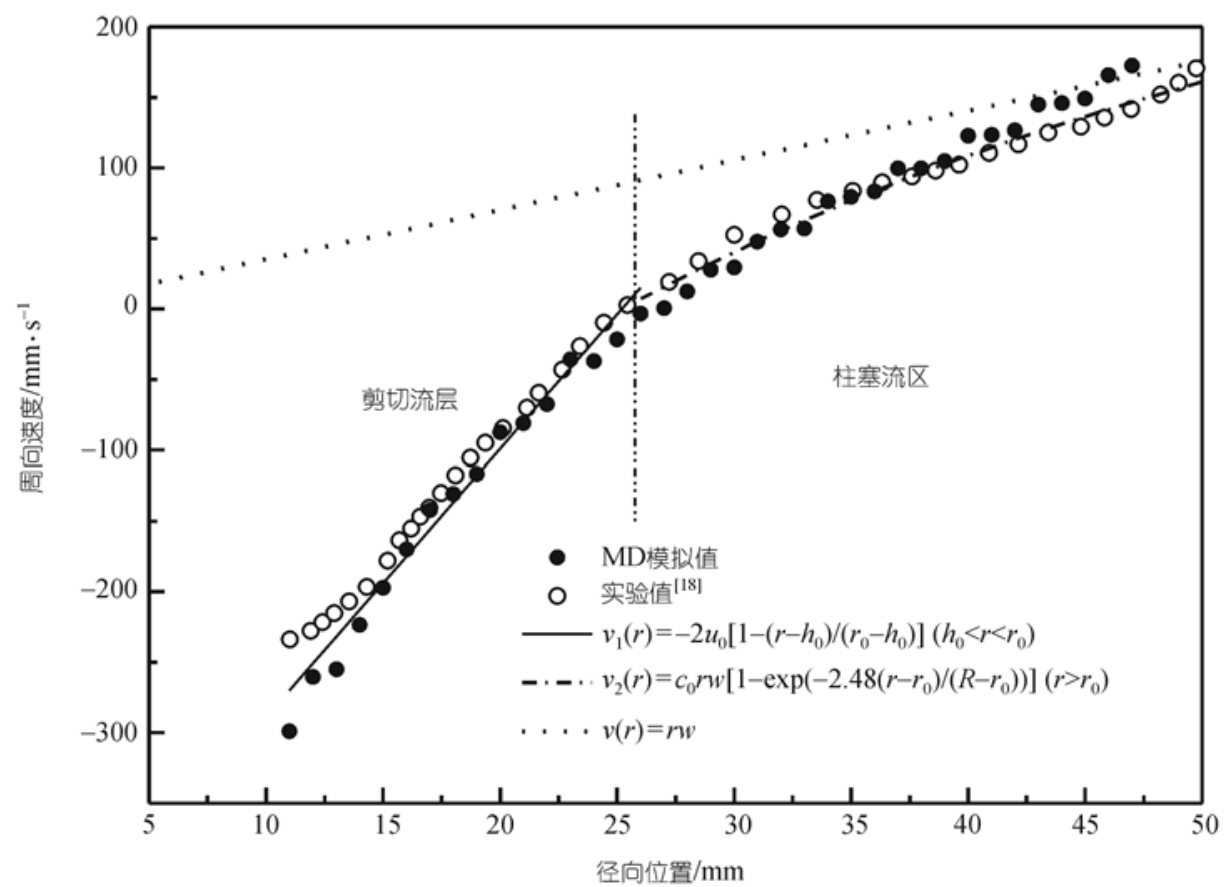

图 7 对称线速度分布实验值、MD模拟值和简化分析值的比较 $(42 \mathrm{r} / \mathrm{min}$ ) 


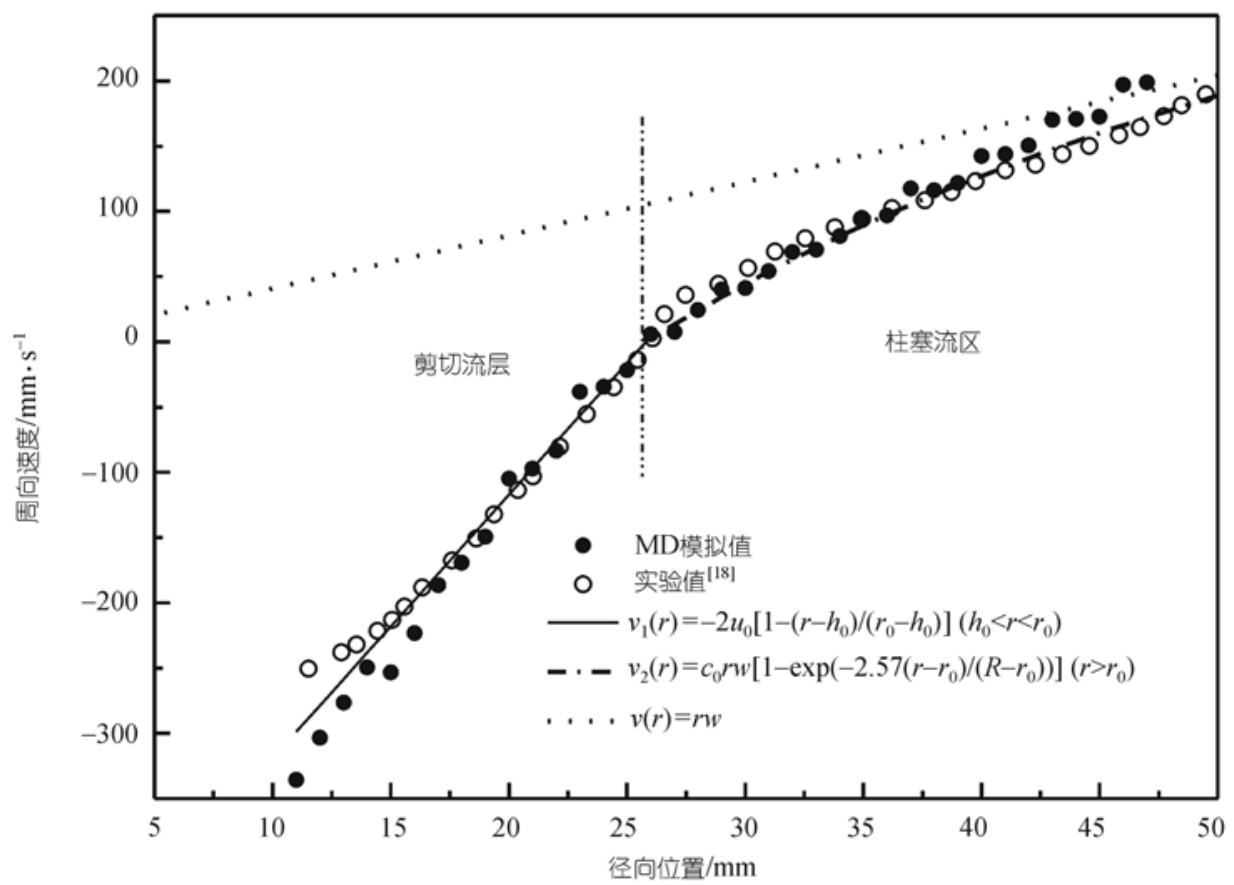

图 8 对称线速度分布实验值、MD 模拟值和简化分析值的比较 $(65 \mathrm{r} / \mathrm{min})$

正至为关键，若无该项修正，颗粒转动角速度随时间 逐渐累积, 运行时间超过一定值之后稳态颗粒流将 无法继续维持. 过去 10 年关于回转筒颗粒流MD模拟 不少 ${ }^{[13,25,27]}$, 但能对中线速度进行验证的却不多, 除 本文滚动摩阻采用 $\bar{\omega}_{i}$ 一阶修正外, Yang等人 [25]零阶 修正亦可参考, 但真正物理机制仍有待研究.

\section{3 对称线上速度分布的连续理论分析}

图 7 和 8 表明, MD数值实验可以提供准确的颗粒 流场信息, 回转筒反应器的设计还将依赖于从这些 信息中归纳的宏观连续理论. 如图 5 和 6 所示, 对于

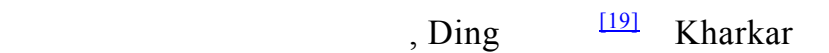
人 ${ }^{[211}$ 和Maneval等人 ${ }^{[26]}$ 在发展连续理论时都采用 $V_{2}(r)=r w$ 的假设, 尽管这些学者都意识到柱塞流区 内不同径向位置颗粒周向速度存在着塑性蠕变所造 成的相对滑移, 即 $r w$ 这种刚性分布曲线并不能准确 描述实际速度分布, 但他们并未总结出更合理的表 达式. 我们经过长期MD模拟结果和实验观察, 认为 柱塞流区内颗粒周向速度的径向变化符合指数衰减 规律:

$$
V_{2}(r)=c_{0} r w\left[1-\exp \left(-m_{0} \frac{r-r_{0}}{R-r_{0}}\right)\right] \quad r_{0}<r<R_{0},
$$

其中 $c_{0}$ 表示筒壁和第一层颗粒速度间滑移比, $m_{0}$ 表
征塑性蠕变而造成的颗粒层刚性转动衰减度. 而对 于活性层的颗粒流动符合快速切变流动, 可采用简 单的 Couette 流动分布描述:

$$
V_{1}(r)=-2 \bar{u}_{0}\left(1-\frac{r-h_{0}}{r_{0}-h_{0}}\right) \quad h_{0}<r<r_{0},
$$

$c_{0}$ 和 $m_{0}$ 值可由 MD 模拟或实验结果根据(16)式拟合 得到, 则 $V_{2}(r)$ 速度分布已知后, (17)式中活性层平均 速度 $\bar{u}_{0}$ 可由对称线上质量守恒式(18)计算, $\bar{u}_{0}$ 求解 后则 $V_{1}(r)$ 也可得:

$$
\int_{h_{0}}^{r_{0}} V_{1}(r) \mathrm{d} r=\bar{u}_{0}\left(r_{0}-h_{0}\right)=-\int_{r_{0}}^{R} V_{2}(r) \mathrm{d} r .
$$

由图 7 和 8 , 两种转速下(17)和(18)式理论分析都 可准确地反映对称线上速度分布. $42 \mathrm{r} / \mathrm{min}$ 时筒壁处 速度滑移比 $c_{0}$ 约为 0.8 , 而 $65 \mathrm{r} / \mathrm{min}$ 时为 0.6 , Maneval 等人 ${ }^{[26]}$ 和 Ding等人 ${ }^{[19]}$ 的结果显示, 在 $F r$ 较小的 Rolling模式下 $c_{0}$ 接近 1.0, 因此可以得出中高转速下 回转筒壁和颗粒层将发生明显的速度滑移. 如图 7 下 部柱塞区颗粒速度并非符合 $r w$ 或 $c_{0} r w ，$ 即保持其刚 性, 而是一个典型的蠕变流动, 其中 $42 \mathrm{r} / \mathrm{min} 工$ 况下 $m_{0}=2.48$, 而 $65 \mathrm{r} / \mathrm{min}$ 时 $m_{0}=2.57$, 拟合相关性超过 $95 \%$ ．(16) (18)式计算的速度分布完全符合MD模拟 值和PEPT实验值, 这是已有理论分析未能实现的. 随着今后数值或实物实验进一步开展, (16) (18)式有 
望对已发表连续理论进行修订 [19,21,22].

\section{4 颗粒温度和颗粒相对浓度分布特性}

MD 模拟可提供以往实物实验所无法提供的信 息，如颗粒温度的分布. 颗粒温度反映的是颗粒速度 脉动的强弱, 具体表示为 $\bar{\theta}=\left[\Sigma_{n}\left(v_{i}-\bar{v}\right)^{2} / 3\right] / n$. 图 9 即为颗粒温度分布图, 上部活性层内颗粒温度要远 高于下部柱塞流区, 此外颗粒温度分布沿着对称线 并不对称, 也就是沿倾斜床面滚落到最底部处的颗 粒碰撞尤为剧烈, 因此其颗粒温度更大, 该规律已有 文献报道, 而且与我们后续实验的发现十分吻合, 将 另文讨论. 图 10 是二维床颗粒相对浓度的分布(最大 浓度值设为 1), 我们可以推断出颗粒在下部区域运 动更像一个个随筒体转动的流束，尽管不同径向位 置流束角速度有衰减, 但各个流束可假设为柱塞流. 下部区域中颗粒浓度接近 1.0 的区域反映的是二维床 中颗粒球填充的部分, 而颗粒浓度比较低以至低于 0.1 的区域应该指的是颗粒填充后空隙. 这种颗粒流 动轨迹仅能在二维床 MD 模拟中可以看到, 三维床中 轴向平均的统计将会掩盖这一重要现象.

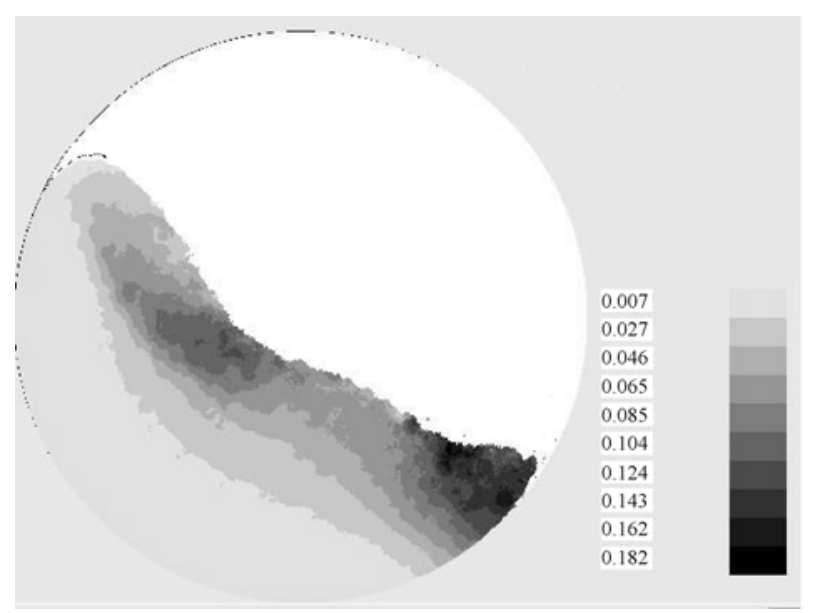

图 9 回转筒内颗粒温度的等势图 $(42 \mathrm{r} / \mathrm{min})$

\section{3 结论}

颗粒模拟和传统分子模拟的不同在于颗粒之间 存在着非弹性接触, 及符合库仑屈服定律的滑动摩 擦和滚动摩擦, 本文综合这些因素拓展了分子动力 学算法, 成功实现了中高速 $(F r=0.1 \sim 0.2)$ 回转筒内颗 粒流的模拟. 结果表明, 筒内颗粒流由表面自由滚落 的活性层和下部柱塞流区组成, 颗粒在活性层的停 留时间约为柱塞流区的 $1 / 3 \sim 1 / 2$, 对称线上活性层和 柱塞流区的厚度比为 $0.57 \sim 0.61$, 因而推断筒内颗粒

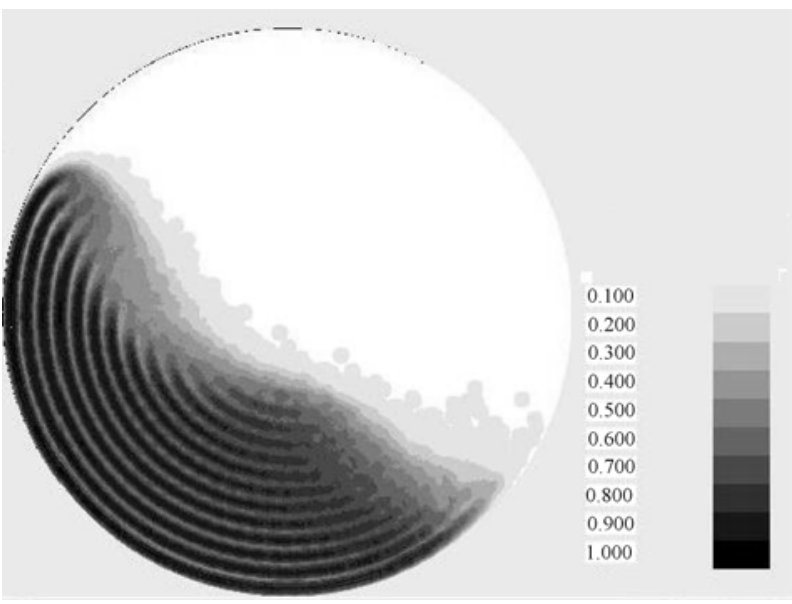

图 10 回转筒内颗粒相对浓度的等势图 $(42 \mathrm{r} / \mathrm{min})$

流动并非处于传统 Rolling 模式, 而应处于 Rolling-Cascading 过渡区. 对称线上 MD 模拟的速度 分布和文献中正电子放射性测量实验结果相当吻合. 模拟和实验结果都揭示了柱塞流区内颗粒运动并非 完全随筒壁做刚体转动, 而是不同径向位置颗粒层 之间存在着塑性蠕变, 这种速度渐变过程符合指数 函数规律; 而活性层内颗粒流动则可视为简单的 Couette 切变流分布. 该模式下颗粒温度分布沿着对 称线并不对称, 亦即颗粒沿倾斜床面滚落到最底部 处的颗粒碰撞尤为剧烈.

致谢 感谢在 Leeds 大学访问时与 Xu B H 博士之间有益的 讨论, 感谢课题研究过程中南威尔士大学 Feng Y Q 博士所 给予支持和帮助. 本工作受国家自然科学基金(批准号: 50306012) 和国家重点基础研究发展计划 (编号: 2002CB211600)资助.

\section{参考文献}

1 Jaeger H M, Nagel S R, Behringer R P. Physics of the granular state. Science, 1992, 255: 1523-1531[DOI]

2 Duran J, Reisinger A, Sands, et al. An Introduction to the Physics of Granular Materials. New York: Springer-Verlag, 2000

3 Hostler S R. Wave propagation in granular materials. Dissertation for the Doctoral Degree. California Institute of Technology at Blvd Pasadena, 2005, 1-15

4 Zhou Y C, Xu B H, Yu A B. Numerical investigation of the angle of repose of monosized spheres. Phys Rev E, 2001, 64: 021301[DOI]

5 Johnson P C, Nott P, Jackson R. Frictional-collisional equations of motion for particulate flows and their application to chutes. J Fluid Mech, 1990, 210: 501-535[DOI]

i6 CHunt M L, Weathers R C, Lee A T, et al. Effects of horizontal vi- 
bration on hopper flows of granular materials. Phys Fluids, 1999, 11: $68-75[\mathrm{DOI}]$

7 Li S Q, Yan J H, Li R D, et al. Axial transport and residence time of MSW in rotary kilns: Part I. Experimental. Powder Tech, 2002, 126: $217-227[\mathrm{DOI}]$

8 Metcalfe G, Shinbrot T, McCarthy J J, et al. Avalanche mixing of granular solids. Nature, 1995, 374: 39—41[DOI]

9 Shinbrot T, Alexander A, Muzzio F J. Spontaneous chaotic granular mixing. Nature, 1997, 397: 675-678

10 Rapaport D C. The Art of Molecular Dynamics Simulation. 2nd ed. London: Cambridge University Press, 2004

11 葛蔚, 麻景森, 张家元, 等. 复杂流动多尺度的粒子方法. 科学 通报, 2005, 50(9): 841-853

12 Dury C M, Ristow G H. Competition of mixing and segregation in rotating cylinders. Phys Fluids, 1999, 11: 1387-1394[DOI]

13 Dziugys A, Peters B. An approach to simulate the motion of spherical and non-spherical fuel particles in combustion chambers. Granular Matter, 2001, 3: 231-265[DOI]

14 Brilliantov N V, Pöeschel T. Rolling friction of a viscous on a hard plane. Europhys Lett,1998, 42: 511-516[DOI]

15 Goldenberg C, Goldhirsch I. Friction enhances elasticity in granular solids. Nature, 2005, 435: 188-191[DOI]

16 Weber M W. Simulation of cohesive particle flows in granular and gas-solid system. Dissertation for the Doctoral Degree. University of Colorado at Boulder, 2004, 73-108

17 Stevens A B, Hrenya C M. Comparison of soft-sphere models to measurements of collision properties during normal impacts. Powder Tech, 2005, 154: 99-109[DOI]

18 Parker D J, Dijkstra A E, Martin T W, et al. Positro emission particle tracking studies of spherical particle motion in rotating drums. Chem Eng Sci, 1997, 52: 2011-2022[DOI]
19 Ding Y L, Seville J P K, Forster R, et al. Solid motion in rolling mode rotating drums operated at low to medium rotational speeds. Chem Eng Sci, 2001, 56: 1769-1780[DOI]

$20 \mathrm{Xu}$ B H, Yu A B. Numerical simulation of gas-solid flow in a fluidized bed by combining discrete particle method with computational fluid dynamics. Chem Eng Sci, 1997, 52: 2785-2809[DOI]

21 Khakhar D V, McCarthy J J, Shinbrot T, et al. Transverse flow and mixing of granular materials in a rotating cylinder. Phys Fluids, 1997, 9: 31-43[DOI]

22 Boateng A A. Boundary layer modeling of granular flow in the transverse plane of a partially filled rotating cylinder. Int J Multiph Flow, 1998, 24: 499-521[DOI]

23 Mellmann J. The transverse motion of solids in rotating cylinders_- forms of motion and transition behavior. Powder Tech, 2001, 118: 251-270[DOI]

24 Orpe A, Kharkar D V. Scaling relations for granular flow in quasi-two-dimensional rotating cylinders. Phys Rev E, 2001, 64, 031302[DOI]

25 Yang R Y, Zou R P, Yu A B. Microdynamics analysis of particle flow in a horizontal rotating drum. Powder Tech, 2003, 130: 138$146[\mathrm{DOI}]$

26 Maneval J E, Hill K M, Smith B E, et al. Effects of end wall friction in rotating cylinder granular flow experiments. Granul Matt, 2005, 7: 199-202[DOI]

27 Wightman C, Moakher M, MuzzioF J, et al. Simulation of flow and mixing of particles in a rotating and rocking cylinder. AIChE, 1998, 44: 1266-1276[DOI]

(2006-01-22 收稿, 2006-08-22 接受) 